Itinéraires Itinéraires

Littérature, textes, cultures

\title{
Écriture du corps dans les romans de ninjas (忍者) de Yamada Fûtarô (山田 風太郎)
}

\section{Michihiro Nagata}

\section{OpenEdition}

\section{Journals}

Édition électronique

URL : http://journals.openedition.org/itineraires/1555

DOI : $10.4000 /$ itineraires. 1555

ISSN : 2427-920X

Éditeur

Pléiade

\section{Édition imprimée}

Date de publication : 1 novembre 2011

Pagination : 141-149

ISBN : 978-2-296-55720-8

ISSN : $2100-1340$

\section{Référence électronique}

Michihiro Nagata, «Écriture du corps dans les romans de ninjas (忍者) de Yamada Fûtarô (山田 風太 郎) », Itinéraires [En ligne], 2011-3 | 2011, mis en ligne le 01 novembre 2011, consulté le 19 avril 2019. URL : http://journals.openedition.org/itineraires/1555; DOI : 10.4000/itineraires. 1555

\section{(c) $\oplus \Theta \Theta$}

Itinéraires est mis à disposition selon les termes de la licence Creative Commons Attribution - Pas d'Utilisation Commerciale - Pas de Modification 4.0 International. 


\title{
Écriture du corps dans les romans de ninjas (忍者) de Yamada Fûtarô (山田 風太郎) ${ }^{1}$
}

\begin{abstract}
Yamada Fûtarô is famous for his Ninja novels. His originality resides in the representation of the human body. Each ninja has an original skill that exploits one's physical characteristic. Most often he seeks to give his romantic inventions some objectivity by using a pseudo-scientific vocabulary. What is fundamental to him is the sagacity of the medical gaze that demystifies the human body as an object. Like a surgeon who operates the body without mixing emotional value, Yamada "sutures" or "transplants" some corporal elements of his characters. In the climate of modern Japanese literature, its excessive aspects make his novels of Ninja stand out particularly.
\end{abstract}

Keywords : ninja, human body, medicine, war, humor

Mots clés : ninja, corps humain, médecine, guerre, humour

\section{Petite biographie de Yamada Fûtarô}

Yamada Fûtarô (1922-2001) est un romancier exemplaire, né à Hyôgo où l'on a ouvert récemment un musée en son honneur, à l'emplacement de son ancienne école. Fils d'une famille de médecins, sa première intention fut de devenir lui aussi médecin, conformément à la tradition familiale. En effet, alors qu'il suivait les cours du Tôkyô Medical College, actuellement Tôkyô Medical University, son premier roman ${ }^{2}$ remporta un prix décerné par un magazine de romans policiers. Après avoir obtenu son diplôme de médecine générale en 1950, il décida de devenir romancier. Yamada Futarô doit son triomphe notamment aux récits de ninjas (Les Parchemins

1. Le présent article doit une de ses sources d'inspiration à la postface par Kiyoshi Kasai à Kenki Ramabustu de Yamada Fûtarô dans l'édition Kodansha Novels Special (1994).

2. Darumatouge no Jiken (達磨峠の事件), revue Hôseki (janvier 1947). 
Ninpô) qui ont été largement adaptés au cinéma et en bandes dessinées. Si son dernier roman remonte à 1991, Yamada Fûtarô est resté un auteur très populaire.

\section{Qui sont les ninjas?}

Les ninjas (忍者) sont des guerriers-espions, dont le rôle fut important au Japon à l'époque féodale. Ils portent une tenue serrée au corps, des jambières, et des tabi (des chaussures plates et légères). Le visage voilé, ces soldats clandestins doivent être experts dans les techniques spéciales de combat et surtout de camouflage (Ninpô, 忍法 ou Ninjutsu, 忍術), transmises en secret par leurs ancêtres, afin d'accomplir des missions dangereuses. Le terme Nin (忍) ou son équivalent Shinobi (忍び) désignent 1'art (jutsu, 術) de se dissimuler. Le terme kunoichi est utilisé spécialement pour une femme ninja suivant une lecture particulière des trois traits $(<\zeta 一)$ qui servent à tracer le kanji de la femme en japonais (女).

D'origine très humble, les ninjas furent utilisés à partir de la fin du $\mathrm{XV}^{\mathrm{e}}$ siècle par les daimyôs (seigneurs féodaux) pour assassiner leurs ennemis ou pénétrer dans leurs forteresses. Ces guerriers marginaux et difficiles à inféoder à un seigneur déterminé eurent deux clans principaux, formés dans les régions d'Iga et Koga. Par là, suivant leur provenance géographique, les ninjas étaient souvent nommés hommes d'Iga (Iga mono) ou de Koga (Koga mono). Mais, leur existence resta longtemps dans l'ombre et ils n'apparurent au premier plan de l'histoire qu'avec l'avènement des Tokugawa en 1603. Au cours de la longue période de la Pax Tokugawa, leur efficacité fut moindre, et les deux clans d'Iga et de Koga, sous le contrôle du gouvernement de Tokugawa, se chargeaient de surveiller le château du shogun et de maintenir l'ordre public de la capitale Edo. Dans le Japon moderne, dès l'ère Meiji, ils ne correspondaient plus à aucun rôle social, et le Ninjutsu fut officiellement interdit.

\section{Représentations du ninja}

Depuis le deuxième tiers de l'époque d'Edo (XVIII siècle), ces guerriers de l'ombre alimentaient l'imaginaire du peuple dans le kabuki, dans lequel ils étaient souvent dotés de pouvoirs surnaturels et apparaissaient comme héros invincibles. Jiraiya est l'un de ces personnages de kabuki les plus connus. Il sait manier la magie, et se transforme même en crapaud. À l'ère de Meiji, une telle image du ninja-surhomme nourrissait le kodan, style de conte oral, et sa version écrite, la « Bibliothèque de Tatsukawa », connut un énorme succès.

Le Japon d'après-guerre vit l'épanouissement imaginaire des ninjas dans l'univers des mangas, des films et de quelques romans (on devrait y ajouter les jeux vidéo aujourd'hui). Seulement, les ninjas n'étaient plus décrits comme des personnages fantastiques, mais ils représentaient plutôt 
un peuple aliéné qui combat dans l'ombre pour une autorité à laquelle ils sont destinés à se soumettre en tout. L'organisation hiérarchique des clans ne permet pas à un ninja de rang inférieur d'avoir un nom, ni un titre, ni une place dans la société. Shinobi no Mono, roman de Murayama Tomoyoshi ${ }^{3}$, fit apparaître ces ninjas maniant diverses techniques avancées, qui constituent une sorte de figure allégorique des salariés japonais des années 1960 se consacrant entièrement à l'entreprise. L'image du ninja comme un être de l'ombre se réitère dans Iga no kagemaru de Yokoyama Mitsuteru ${ }^{4}$, un chefd'œuvre monumental du manga. La série des Parchemins Ninpô de Yamada Fûtarô, qui a probablement influencé ces ouvrages contemporains, ne fait pas exception à l'égard de la façon de décrire les personnages de ninjas.

\section{Travestissement inhérent à la pratique d'adaptation}

Afin d'aborder le thème de l'écriture du corps chez Yamada Fûtarô, nous voudrions commencer par un film « d'après Yamada Fûtarô », intitulé Shinobi ${ }^{5}$. Voici le résumé du film : au XVII siècle, le Japon féodal est dirigé par la famille Tokugawa qui a su unifier le pays en imposant la paix. Pour en finir définitivement avec le chaos, le shogun décide de mettre un terme à la rivalité existant entre les deux grands groupes de ninjas, Iga et Koga. Profitant de la haine ancestrale existant entre les deux familles, sous prétexte de désigner l'héritier du royaume, le Shogun ordonne aux chefs des deux clans, affiliés respectivement à un candidat du futur shogun, de faire un combat avec leurs dix meilleurs guerriers. Là, les ninjas se trouvent ballottés par un pouvoir arbitraire.

Or, le film se focalise sur le sort d'Oboro et de Gennosuke, tous deux amoureux l'un de l'autre et héritiers des deux familles Iga et Koga, qui devront choisir entre leur amour ou la loyauté à leur clan. Le combat se termine par l'élimination des uns par les autres, Oboro et Gennosuke ne pouvant pas échapper non plus à ce destin maudit.

C'est un " Roméo et Juliette », certes, faisant le récit d'un amour tragique de deux jeunes gens étant le jouet de l'inimitié qui oppose leurs deux maisons. Mais, l'essentiel du texte original de Yamada Fûtarô (Kôga Ninpô-chô ${ }^{6}$ ) n'existe pas dans cette intrigue shakespearienne.

3. Tomoyoshi Murayama (1901-1977), écrivain, dramaturge et producteur de pièces de théâtre. Son roman Shinobi no Mono est paru entre 1960-1962 dans le journal communiste Drapeau Rouge.

4. Iga no kagemaru est prépublié dans le magazine Weekly Shônen Sunday entre 1961 et 1966.

5. Produit par la société de production cinématographique Shôchiku (松竹), 2005. Le film a été distribué en salle en 2007.

6. Voir le premier tome du Parchemin Ninpô. 


\section{Corps monstrueux des ninjas et kunoichi}

L' « anormalité » ou la « monstruosité » du corps humain est un élément qui revient fréquemment chez Yamada Fûtarô. Chaque ninja possède une technique originale qui exploite sa particularité corporelle, et qu'il arrive à maîtriser après des années d'entraînement : Amayo Jingorô transforme son corps pour prendre la forme d'une limace afin de s'approcher en toute discrétion et d'attaquer son adversaire par derrière; Akeginu, faisant sortir du sang par tous les pores, répand un brouillard rouge mortel; Jimushi Jube, sans bras ni jambes peut ramper, telle une chenille, ventre à terre grâce à des muscles intercostaux particulièrement développés, etc.

Ces experts s'affrontent sous forme de combat par équipe. Les épreuves de cette compétition continuent jusqu'à la mort d'un des participants. Ainsi, le résultat d'une rencontre influence inévitablement la suivante. Cela conduit constamment chaque groupe à élaborer des stratagèmes pour annuler la performance d'un ennemi pivot et avoir l'avantage sur l'adversaire. L'intrigue tragique des deux amoureux recule complètement devant le tournoi de technologies corporelles.

Les innombrables ninjas et kunoichi créés par Yamada Fûtarô ${ }^{7}$ arrivent par un entraînement pénible à développer démesurément la fonction d'un organe particulier de leur corps, pour être capables de répondre à toutes sortes de missions. Ils étonnent le lecteur par leur art secret de ninja ne surpassant pas la limite des possibilités physiologiques mais s'écartant largement du bon sens. Ces arts secrets portent souvent sur le sexe : une kunoichi ${ }^{8}$ transporte dans sa main un organe masculin encore vivant pour le mettre dans le corps d'une femme qui concevra un enfant; un ninja s'arme de son sperme extrêmement adhésif qui, se desséchant, obtient la dureté même du cuir; un ninja et une kunoichi, qui s'enlacent nus tous les deux, se terrent en perçant la terre comme une drille et surprennent l'ennemi qui surgit avec un coup de sabre vertical porté de bas en haut. Marc Kober fait référence à un film, qui est une variante cinématographique de l'art sexuel des kunoichi :

Kunoichi : lady Ninja, film d'Ozawa Hitoshi, avec notamment Moriyama Yuko, est un remarquable film de vengeance en série tourné en 1998. Sept nonnes décident de prendre leur revanche après l'attaque de leur couvent en devenant des ninjas. Elles pratiquent des techniques stupéfiantes, comme l'onde de choc du mamelon de sein (nipple shock wave). Les bouts de leurs seins deviennent lumineux, et elles pratiquent de façon peu violente le vortex vaginal en tourbillon. L'entrejambes de l'une d'entre elles aspire une « powerball » qui est expulsée par la bouche et explose sur le vilain. Ce procédé est dit « Tornado crotch » (la tornade de l'entre cuisses). Le film

7. Outre le Parchemin Ninpô, il a écrit plus de 90 nouvelles de ninjas.

8. Pour une analyse historique et cinématographique des ninjas féminins, voir l'article de Marc Kober, "Les ninjas féminins », Supérieur inconnu, n 3, nouvelle série, 2006, p. 43-48, et notamment p. 48 . 
montre de nombreuses décapitations, ou des corps qui explosent; des larmes peuvent se transformer en billes, puis en épées. Une technique étonnante est celle de l'écho qui revient : l'énergie est aspirée par le vagin, puis recrachée sur l'ennemi. La technique dite de « Virgin blood attack » est assez inédite. En somme, nous avons affaire à une extrapolation du rôle séducteur de la kunoichi, qui utilise le désir sexuel comme une arme, jouant de leur faiblesse pour être invincibles. Et cette riche invention d'un nouvel arsenal issu des organes sexuels, ou des yeux de la combattante, n'est pas en désaccord avec l'inventivité des premiers ninjas 9 .

S'agissant de ces corps anormaux, il n'est pas inconvenant d'évoquer l'existence des phénomènes de l'époque pré-moderne, ces corps monstrueux qui mêlaient horrible et merveilleux, suscitant autant l'attirance que la répulsion. Mais, avec l'avènement d'une société moderne, le corps exceptionnel, enfermé de plus en plus dans le discours médical, constitue le lieu d'émergence des pathologies. Ce qui est intéressant, c'est qu'un écrivain et médecin comme Yamada Fûtarô a fait renaître ces corps considérés comme « hors-norme » dans le monde moderne. Ce thème fera l'objet d'une de nos recherches futures.

De nombreux films basés sur les romans de Yamada Fûtarô, notamment une série de films Kunoichi Nimpochô (la société de production King Records) dans les années $1990^{10}$, ont tendance à mettre en valeur l'aspect sensuel et la dimension fantastique du monde romanesque de Yamada Fûtarô.

\section{Hyper-technologie du corps}

Pur produit de l'imagination, certes, le corps imaginé par Yamada Fûtarô ne s'égare pourtant pas dans un imaginaire aberrant; il cherche le plus souvent à donner à ses inventions romanesques une certaine objectivité en recourant à un vocabulaire pseudoscientifique, si bien que les Ninpôs (techniques des ninjas) prennent l'apparence d'une « hyper-technologie du corps».

Ainsi par exemple, dans un passage de la nouvelle Mushi no Nimpôchô (L'Art de la semence), Hidetsugu, fils adoptif du grand shogun Hideyoshi, maître du Japon avant le règne des Tokugawa, accusé de conspiration contre son père, attend de lui l'ordre de se faire seppukku (c'est-à-dire de s'ouvrir le ventre); juste après avoir été convoqué par son père pour son dernier voyage, Hidetsugu appelle Oasa, kunoichi du clan Kôga, pour lui confier un plan stupéfiant. Oasa est capable non seulement de garder la semence éjectée dans son utérus pendant dix mois, mais aussi de la transmettre dans le corps d'un autre homme avec lequel elle fera l'amour. Ainsi, à travers

9. Marc Kober, op. cit., p. 48. L'article constitue une analyse historique et cinématographique de la figure de la kunoichi.

10. La première vogue de ninja au cinéma date des années 1960 , pendant lesquelles la société de production Tôei (東映) produit des films adaptés à partir des romans de Yamada Fûtarô. 
Oasa, la semence de X est transmise au corps d'un individu Y, et si Y couche avec une autre femme, c'est de la semence de X que Y la gratifiera. Hidetsugu, à l'aide de cet art de la semence d'Oasa, veut se venger de son père en le rendant un simple transmetteur de sa semence et faisant un enfant à la femme de son père.

Dans ce passage, nous voudrions indiquer, en soulignant quelques termes, la maîtrise avec laquelle l'auteur exploite une invention inouïe afin d'éviter de tomber dans l'imaginaire pornographique :

Or, si Oasa était ninja, c'est parce qu'elle avait acquis la connaissance d'un art secret transmis par le clan Kôga, qu'elle était la seule ninja à avoir maîtrisé. Celui-ci consistait à garder les spermatozoïdes dans son utérus. En général, les spermatozoïdes ne peuvent vivre que douze heures dans le vagin, de trois à huit jours dans l'utérus, et quatre heures dans les trompes utérines à cause de l'action anti-microbienne des globules blancs... mais, elle pouvait les conserver en vie pendant dix mois et au cours de cette période, elle pouvait tomber enceinte à son gré ${ }^{11}$.

L'utilisation de termes médicaux ou anatomiques semble pour le moins déconcertante, si l'on tient compte du fait que c'est une histoire qui se déroule dans le Japon féodal. Le plus souvent, Yamada Fûtarô utilise sans aucune réticence un vocabulaire moderne, y compris des termes étrangers tels que « hystérie », « poste de radar », « mètre », peu importe qu'il s'agisse d'un roman de cape et d'épée ou un roman policier se déroulant à diverses époques historiques du Japon. Ce genre d'anachronismes produit un effet humoristique, qui est caractéristique de son style.

\section{Poésie et médecine}

La particularité de l'œuvre de Yamada Fûtarô ne se limite pas à son écriture humoristique. Ce qui est fondamental chez le romancier, c'est avant tout la sagacité du regard médical qui démystifie le corps humain en tant qu'objet. Gérard Danou, dans son ouvrage sur l'interférence entre littérature et médecine, écrit que le langage médical étant toujours neutre, son utilisation dans un texte littéraire empêche l'expression émotionnelle spontanée par effet de rupture et d'étrangeté ${ }^{12}$. En outre, la précision propre à ce métier introduit de la distance par rapport au sujet, d'où il résulte une transformation des valeurs, comme le décrit Victor Segalen dans sa thèse de doctorat en médecine :

Tandis qu'un profane, entrant à l'improviste dans une salle d'opération, raconterait plus tard à ses amis : "C'était épouvantable! Il y avait une pauvre femme renversée, la tête très basse, le ventre ouvert; elle râlait

11. Extrait de « Mushi no Nimpôchô », dans Kunoichi ninpô chôbu [Aspects de l'art des kunoichi], Contes de ninjas, vol. 6, Tôkyô, Chikuma, 2004. Nous soulignons.

12. Gérard Danou, Le Corps souffrant, Seyssel, Champ Vallon, 1994, p. 189. 
continuellement...», le chirurgien dirait plus techniquement : «Je viens d'opérer un fibrome qui m'a donné pas mal de tracas. J'avais mis la malade en position de Trendelenburg. La chloroformisation a été délicate ${ }^{13} \ldots »$.

Les femmes ninjas de Yamada Fûtarô utilisent le désir sexuel et leur séduction comme une arme épouvantable, mais l'auteur cherche à désexualiser leur corps en recourant systématiquement à des descriptions médicales dans son texte. Cette réduction de l'aspect émotionnel par l'intellectualisation du langage constitue une différence déterminante entre les ouvrages romanesques de Fûtarô Yamada et leurs adaptations filmiques ou leurs versions manga.

On peut même remarquer une relation privilégiée à la médecine et à la poésie chez Yamada Futârô. À l'image du chirurgien qui opère le corps sans y mêler aucune valeur émotionnelle, Yamada « suture » ou " greffe » certains éléments corporels de ses personnages. Cette manipulation objective lui permet de concevoir un fil de trame absolument inédit. Par exemple, l'auteur raccorde les vaisseaux de deux êtres humains, afin de s'inventer une histoire dans laquelle une kunoichi, pour se défendre, unit son artère utérine à l'artère du pénis en érection d'un ennemi; ce dernier ne peut la tuer sans courir le risque de perdre du sang (les veines du pénis se resserrent lors de l'érection, la circulation sanguine doit alors passer par l'intérieur de l'utérus de la kunoichi).

\section{Expérience de la guerre}

Débarrasser le corps de toute conception idéaliste et déformer ce corps ou le morceler en éléments anatomiques selon une nécessité narrative. Ce sont les écrivains ayant eu l'expérience d'études médicales qui excellent en une telle manière d'écrire comme par exemple Abe Kôbô ${ }^{14}$, qui écrit un récit (La Face d'un autre) : un homme défiguré par une brûlure au visage se fabrique un masque réaliste mais différent de son visage original; il tente, avec ce nouveau visage, de reconquérir sa femme.

Il est incontestable que Yamada Fûtarô suit les traces de cet écrivain médecin. Toutefois, on peut se demander si cette conception « matérialiste » des corps propre à Yamada provient uniquement de son expérience de médecine. À cet égard, nous voudrions examiner la possibilité de l'influence de la guerre dans sa création romanesque.

La formation de la société japonaise de l'après-guerre présuppose la perte de la Seconde Guerre mondiale. Durant cette guerre, les Japonais ont

13. Cité par Gérard Danou, op. cit., p. 187.

14. Abe Kôbô (1924-1993), écrivain japonais mais aussi militant communiste, fit des études de médecine à l'exemple de son père qui travailla en tant que médecin en Mandchourie. En 1962, son roman La Femme des sables obtint en France le prix du « meilleur livre étranger ». 
subit des expériences cruelles et inhumaines : comme le décrit Sakaguchi Ango ${ }^{15}$, un tas de cadavres carbonisés s'est formé dans les villes dévastées sous les bombardements américains; de nombreux soldats démobilisés et autres victimes aux membres amputés rôdaient dans la cité; partout, des prostituées qui utilisaient leur corps comme seul instrument de survie. Pouvons-nous dire que Yamada Fûtarô a voulu représenter dans ses récits de ninjas ces spectacles horribles que la société japonaise d'après-guerre cherchait à dissimuler et à oublier? Ses romans de combat font apparaître de manière récurrente plusieurs groupes antagonistes qui finissent toujours par s'anéantir et on peut y voir une allégorie de l'hécatombe de la guerre moderne; le corps monstrueux des ninjas et leur instrumentalisation qui démythifie le sexe même semble faire allusion à l'existence de ces rejetons grotesques de la guerre.

Là, Yamada Fûtarô se situe très loin de l'esprit pacifiste, et soucieux de bien-être matériel, de la société d'après-guerre qui bénéfice d'une prospérité sans précédent. Ce genre d'humanisme critique le système social qui a engendré la guerre, mais sa confiance en l'homme n'est jamais ébranlée. Au contraire, le corps grotesque mais aussi extraordinaire des personnages de Yamada Fûtarô nous conduit aux limites mêmes de ce que nous considérons comme humain.

Sur ce point, citons son journal intime écrit à Tôkyô sous les bombes pendant la Seconde Guerre mondiale, journal intime (Le Journal d'un moins que rien) où la critique, par son anti-humanisme moderne, s'avère plus radicale que celle des humanistes modérés et « sensés ». Le 19 avril 1943, il écrit : «La guerre profane la mort. La mort en trop grande quantité prive les gens de la dignité de leur propre mort. En effet, en apparence, ils font l'éloge d'une mort brillante ou d'un sacrifice honorable, mais leur esprit, habitué à la mort, ne voit plus les vraies peur et dignité de celle-ci ${ }^{16}$. $\gg$ Cette mort privée de sa dignité s'impose de manière cruciale dans la description suivante de Tôkyô réduite à l'état de ruines par les bombes incendiaires :

On voyait des corps calcinés partout comme on voit les lézards les jours d'été. Sous le corps carbonisé de la mère se trouvait le corps calciné de l'enfant. [...] Poursuivi par les flammes les gens se jetaient dans la rivière Sumidagawa, mais celle-ci était aussi en feu. Les bateaux étaient incendiés par la pluie de feu, l'eau bouillait. D'innombrables gens sont morts là. Aujourd'hui encore, les cadavres se frottent comme des thons en nombre infini.

Confronté à tant de meurtres insignifiants, l'auteur est devenu indifférent face à la mort violente. De même, les héros et hérö̈nes de Yamada

15. Sakaguchi Ango (1906-1955) est un écrivain japonais qui appartient à la génération précédente de Yamada Fûtarô. C'est son essai sur sa vision de la guerre (Darakuron [ $\mathrm{La}$ Chute], 1946) qui lui a valu sa renommée actuelle. Voir : «La Chute », dans Cent ans de pensée au Japon, t. I, Arles, Picquier, 1996.

16. Yamada Fûtarô, Le Journal d'un moins que rien, Tôkyô, Chikuma, 1998. 
Fûtarô, devant les cadavres de leurs camarades tués comme des bêtes, ne sont jamais saisis par le scepticisme ou le dégoût de soi, sentiments que l' « homme moderne » est censé avoir normalement dans une situation pareille. Les ninjas et kunoichi n'ont pas la moindre inquiétude humaniste. Leur flegme imperturbable est vraiment étonnant.

\section{En guise de conclusion : l'humour de Fûtarô Yamada}

Cependant, les ninjas et kunoichis de Yamada Fûtarô ne plongent pas dans le pessimisme total. Tout en ayant confiance en leur propre technique, ils se jettent dans un combat acharné, et finissent par tous s'entretuer. Ils acceptent résolument la mort comme destin sans jamais montrer un air douloureux. On dirait qu'ils incarnent l'éthique proposée par Sakaguchi Ango : « Suivre la voie de la chute jusqu'au bout, c'est le seul moyen de se découvrir et de sauver soi-même. » L'expérience grotesque de la guerre permet à Yamada Fûtarô de découvrir une autre humanité que celle de l'époque moderne, humanité « monstrueuse » représentée par ces jeunes filles qui jouent sur les lieux de l'incendie :

Mais les petites filles gloussaient. La lumière d'un printemps précoce convenait parfaitement à ce lieu. Alors qu'une victime entrait, ces petites filles se sont levées et d'une voix douce comme si la victime était navrée, elles se sont précipitées en disant «Entrez », mais figées dans leur sentiment d'inertie, elles ne pouvaient pas effacer les traces de leur rire d'avant ${ }^{17}$.

Si ces filles se mettent à rire dans une telle situation dramatique, ce n'est pas parce que leur raison s'est effondrée devant un tas de corps calcinés. Ce rire est peut-être analogique avec un rire grotesque causé par le renversement de l'ordre existant. C'est le rire du monde carnavalesque où la permutation sarcastique des rapports hiérarchiques produit une effervescence collective et un rire éclatant. De la sorte, tout en choisissant la sexualité comme matériau romanesque, les œuvres de Yamada Fûtarô provoquent l'hilarité du lecteur.

Dans le climat de la littérature japonaise moderne où se développe une forme romanesque confidentielle ${ }^{18}$, c'est par ces aspects comiques que ses romans de ninjas se font remarquer singulièrement.

Michihiro Nagata

Université d'Art et de Culture d'Oita (Japon)

17. Ibid.

18. Le genre du roman appelé shi-shōsetsu a pour personnage principal l'écrivain lui-même, avouant les expériences signifiantes de sa propre vie. 